\title{
Radio Transmission and Solar Activity*
}

\author{
By Prof. E. V. Appleton, F.R.S.
}

I PROPOSE to review very briefly the progress made in certain branches of radio research since the last General Assembly of the International Scientific Radio Union in 1934. In selecting such topics for consideration I have been constantly struck, as many others doubtless have been, by the way in which the range of the interests of the radio worker has been gradually expanding, so that now it overlaps almost every field of physical knowledge. In that everyday tool we employ, the amplifying valve, we find problems of thermionics, electrostatics and electrodynamics. The study of aerials and their radiative properties involves problems entirely analogous to those of physical optics. The investigation of the travel of radio waves excites our interest in fundamental atomic processes by way of which the electron population in the ionosphere increases and decreases. The profound control maintained by the sun on the ionosphere and, especially, the response of upper atmospheric conditions to events on the sun's surface, identifies our interests closely with those of the solar physicist, while, by way of the troposphere, which is the seat of atmospheric disturbances, we find much of common concern with the meteorologist.

Radio researches depend fundamentally on the behaviour of the electrical circuits we employ at both sending and receiving stations. To make measurements on long waves and on low-frequency oscillations is comparatively easy, but, with ultrahigh frequency oscillations, where the wave-length is comparable with the size of the apparatus, such measurements have demanded the development of a new technique of great elegance in which our Italian colleagues have played an outstanding part. The result is that, with the availability of new valves and new circuits, a rich field of research has been opened for our exploration.

The study of the travel of ultra-short waves to distances beyond the horizon has again directed attention to the classical problem of the transmission of waves around an imperfectly conducting earth. The pioneer work of G. N. Watson on this subject has been used as the starting point of more recent investigations, but, while the solution of the problem reached by him was formally complete, its application to practical cases has encountered considerable practical difficulties. Elucidations and extensions of Watson's analysis have been

* From the presidential address to the International Scientific Radio Union, delivered in Venice on September 4. recently made by Eckersley, Wwedensky and van der Pol and Bremmer which readily permit the comparison of theory with experiment in practical cases where the sender and receiver are elevated above the ground. As the last two authors have shown, the theory predicts no marked diminution in the intensity of television signals on wavelengths of the order of 7 metres when the receiver passes beyond the optical horizon of the sender. The attenuation of such signals is, in fact, determined by the finite conductivity of the earth rather than by lack of bending over moderate distances. Whether atmospheric refraction further assists the influence of the diffraction is still not quite certain, and further systematic experimental investigations on the subject are desirable.

The systematic investigation of the ionosphere by means of radio waves has been actively pursued during the last four years. The critical frequency method of measuring such upper-atmosphere ionization densities has now received widespread adoption, and the register compiled by the Ionosphere Sub-Commission shows that as many as twenty-four stations are at present making measurements of this type in different parts of the world. Observations have, for example, been made within ten degrees of the North Pole and within forty-seven degrees of the South Pole. In some cases both sender and receiver are manually operated ; in others, part or all of the apparatus is automatic and requires merely maintenance attention. But in all cases there is obtained the relation between equivalent height of reflection at vertical incidence and electric wave frequency, from the features of which the maximum electron concentrations in the various ionospheric regions may be calculated.

Observations made by the critical frequency method are now sufficiently extensive to permit us to make various deductions concerning the major ionospheric variations. There is now ample evidence to show that, during the daytime, the behaviour of Regions $E$ and $F_{1}$ is such as can be explained by a simple theory involving the simultaneous operation of photo-ionization by solar radiation and recombination. Region $F_{2}$, on the other hand, behaves abnormally, since experiments conducted in the northern hemisphere have shown that, over a period of years, the summer noon ionization is actually less than the corresponding winter value. Valuable additional 
information on this point has more recently resulted from the comparison of results made at the two stations of Washington and Watheroo, which are situated at approximately the same distances north and south of the equator. At first it appeared that Region $F_{2}$ ionization varied similarly at both of these stations, indicating an annual effect, but a more extended series of observations showed that this was not the case. The nature of the anomaly was clearly elucidated by Goodall, who in a very important discussion of the available Washington and Watheroo data, showed that there is a seasonal variation in both hemispheres together with non-cyclic changes which appear to affect both hemispheres similarly and is related in some way to the general solar activity. It will be thus seen that there are two problems associated with Region $\boldsymbol{F}_{2}$. One is to explain the anomalous seasonal effect, first recognized in the northern hemisphere, and the other is to explain the non-seasonal variation which has been found in the examination of the valuable observations in both hemispheres made by Berkner and his colleagues under the auspices of the Department of Terrestrial Magnetism of the Carnegie Institution of Washington.

Advantage has been taken by members of the International Scientific Radio Union to make ionospheric observations on the occasion of three out of the four solar eclipses which have taken place since 1934. The results have amply confirmed the earlier conclusions that the electrons in Regions $E$ and $F_{1}$ are liberated by solar photons and are captured according to a recombination law. On the occasion of the total eclipse in Japan in 1936, an opportunity also arose to investigate the origin of the so-called abnormal or sporadic Region $E$ echoes which appear to be due to locally ionized clouds or strata embedded within the normal Region $E$. From the results obtained there is a suggestion of a partial solar photon origin, but it would perhaps be wiser to regard this more as an incentive to further work on the subject than as a definite conclusion. The extensive results for the same eclipse have, unfortunately, not solved the vexed question of the origin of Region $F_{2}$, for while some observers interpret the data as indicating ionization by photons, others argue, equally plausibly, in favour of ionization by corpuscles. There is, evidently, need for a further critical examination of the available data in the light of the known abnormal behaviour of Region $F_{2}$, as well as for the harvesting of further experimental observations. The forthcoming solar eclipse of October 1, 1940, in South Africa and South America should provide us with an excellent opportunity for the latter. The preparatory calculations concerning both optical and corpuscular eclipses in the ionosphere for this occasion have already been made by H.M. Nautical Almanac Office, Great Britain.

I now turn to a fascinating story in the history of solar physics, the first chapter of which was written many years ago. On September 1, 1859, a historic observation was made by R. C. Carrington, a British astronomer. While making, in the forenoon of that day, his customary examination of the forms and positions of spots on the sun, he was startled to note two patches of intensely bright and white light associated with a particular group. He hastily left his apparatus to call someone else to witness the phenomena with him but, on returning within sixty seconds, was, to use his own words, "mortified to find that the outburst was already much changed and enfeebled". On calling at Kew Observatory a day or two afterwards, Carrington was interested to learn that, at the very moment he had seen the solar flare, the three magnetic elements at Kew were similarly disturbed. About $17 \frac{1}{2}$ hours after these disturbances, there occurred one of the most violent magnetic storms ever observed. It should be here noted that the year 1859 was one of sunspot maximum.

This interesting story has acquired fresh significance in recent years as a result of the fertile collaboration of many workers on the subject of solar and terrestrial relationships. In 1930 Mögel, in an undeservedly neglected paper, analysed a series of short-wave radio observations made during the years of sunspot maximum 1927-29. In this paper he describes the occurrence of what he calls "short-disturbances" or interruptions of radio communication over circuits traversing the illuminated side of the earth. Such short-disturbances were found to affect communication for periods up to an hour and were accompanied by a characteristic disturbance of the earth's magnetic field. Mögel suggested that the radio and magnetic effects were due to abnormal ionization in the lower layers of the atmosphere.

It would, of course, scarcely be permissible to deduce from the single observation of Carrington and the work of Mögel that the three phenomena, solar flare, radio fade-out and characteristic magnetic disturbance, were associated. That connexion has been satisfactorily established only as the result of the combined efforts of many observatories and radio stations. Such collaboration was prompted first by the French National Committee of the International Union which, through its chairman, M. Jouaust, directed the attention of other national committees to the reappearance of Mögel's "short-disturbances" in 1935. From that year onwards an extensive research has been conducted by numerous 
organizations and individuals. At the request of Dr. J. H. Dellinger, for example, special solar observations were made at Mount Wilson Observatory at times when fade-outs were expected, to see if any visible solar effect could be associated with them, while an examination of old spectroheliograms immediately revealed the fact that a number of fade-outs already recorded had been accompanied by bright chromospheric eruptions. But perhaps the first really striking example of these interrelated phenomena occurred on April 8, 1936, when a very intense solar flare photographed at Mount Wilson was found to occur simultaneously with a widespread radio fade-out and the characteristic magnetic variation. As the solar activity has increased in recent years, many more examples of such correlations have been noticed.

To the student of the ionosphere, the temporary abnormal absorption of radio-waves during a fade-out is a phenomenon of great interest. When the disturbance is not too severe, and weak echoes can still be received, it is generally found that the height of reflection is not materially altered. The fade-out must thus be due, as Mögel suggested, to increased ionization at levels below those normally responsible for the reflection of medium and short radio waves. Such increased electron content in what was identified many years ago as Region $D$ would be expected to have three effects. It would temporarily increase the conductivity of the lower ionosphere for the overhead currents which cause the daily magnetic variations, and so account for the observed magnetic disturbance. It would increase the normal Region $D$ attenuation of radio waves passing through it and so explain the occurrence of the short and medium wave fadeout. At the same time, however, it would increase the reflection coefficient of Region $D$ for the very long waves which are deviated by its lower boundary. Such enhanced reflection of very long waves on the occasion of a short-wave fade-out was first noted by $R$. Bureau in his study of the propagational characteristics of atmospherics.

There can be little doubt that the enhanced ionization in Region $D$ during a solar flare is electronic in character, and that the exaggerated attenuating properties of this region as a radio transmission medium is due to the high frequency with which the electrons collide with the neutral molecules. Since the attenuating stratum is produced at a low level, the atmospheric absorption coefficient of the solar photons responsible must be relatively high. Possibly the electrons in question are liberated by photo-detachment from the negative ions which we suspect are present in large numbers during the daytime in Region $D$.

\section{Archæological Sequence in North-West Honduras}

$\mathrm{D}^{2}$ URING 1936 a joint archæological expedition of the Smithsonian Institution and Harvard University excavated a number of sites in the Departments of Cortes, Yoro and Santa Barbara in north-west Honduras. The expedition, which was in the nature of an archæological reconnaissance rather than a systematic exploration, had as its objective in part the extension of the explora. tory work carried out by Dr. W. D. Strong in north-eastern Honduras on behalf of the Smithsonian Institution in 1933, in part, and more immediately, to carry further the work of Byron D. Gordon (1895-97) and of the late Mrs. Dorothy H. Popenoe (1928-29). Mrs. Popenoe's pioneer work at Playa de los Muertos, where Gordon also excavated, in affording valuable and much-needed evidence of the stratigraphical relations of early cultures in this region, had opened up new vistas in the study of the archæology of Honduras. This evidence it was the purpose of the expedition to test and extend.

A detailed report of the results of the excavations of 1936 is in course of preparation; but as the mass of material precludes early publication, a preliminary report has been prepared by Dr. Strong, the senior member of the expedition, and his colleagues, in which an account is given of the major results emerging from the investigation.*

The sites investigated lie in what may be termed the drainage area of the Ulua River (including the Chamelecon River, which formerly drained into it) north-eastward from Lake Yojoa. The environmental conditions of this region belong to two types. On one hand are the broad alluvial valleys of the lower Ulua and Comayagua Rivers with dense rain forests; on the other hand are the elevated mountain valleys with clear flowing streams, in which the rain forest is limited to the border of the stream or, in the Lake Yojoa area, of the lake, while raised plateaux with oak and pine afford a background.

The sites examined are distributed geographic. ally in three groups : the Chamelecon group, of which Naco is the most extensive, the Ulua and Comayagua group, of which Playa de los Muertos

* Preliminary Report on the Smithsonian Institution-Harvard University Archæological Expedition to North-Western Honduras, 1936. By William Duncan Strong, Alfred Kidder II, and A. J. 1936. Brel Paul, Jr. Smithsonian Miscell. Collect., 97, 1, 1938. Pp. 129 Drexel Paul,
+14 plates. 